\title{
Establishment and Characterization of 10 Human Pancreatic Cancer Cell Lines Including a HER2 Over-Expressed Cell Line
}

\author{
SUPPLEMENTAL DIGITAL CONTENT
}

\begin{tabular}{|c|c|c|c|}
\hline \multicolumn{4}{|c|}{ SUPPLEMENTARY TABLE 1. Primer Sequences Used in the Study } \\
\hline Name & Forward Sequence & Reverse Sequence & $\mathbf{T m},{ }^{\circ} \mathrm{C}$ \\
\hline$V E G F-A$ & AGTCCAACATCACCATGCAG & CAAGGCCCACAGGGATTT & 60 \\
\hline$V E G F-C$ & GCCAACCTCAACTCAAGGAC & CCCACATCTGTAGACGGACA & 60 \\
\hline$H I F-1 a$ & ATGGAGGGCGCCGGCGGCGCGAACGA & AATCATGTCACCATCATCTGTGAGAA & 65 \\
\hline Cyclin A & CTGGACCCAGAAAACCATTG & CCTCTCAGCACTGACATGGA & 59 \\
\hline Cyclin B1 & TGTGGATGCAGAAGATGGAG & AAACATGGCAGTGACACCAA & 59 \\
\hline Cyclin D & GTGGCCTCTAAGATGAAGGAG & GAACTTCACATCTGTGGCACAG & 60 \\
\hline Slug & GGGGAGAAGCCTTTTTCTTG & TCCTCATGTTTGTGCAGGAG & 58 \\
\hline E-Cadherin & TGCCCAGAAAATGAAAAAGG & GTGTATGTGGCAATGCGTTC & 59 \\
\hline$N$-Cadherin & ACAGTGGCCACCTACAAAGG & CCGAGATGGGGTTGATAATG & 60 \\
\hline MMP9 & CTCGAACTTTGACAGCGACA & GCCATTCACGTCGTCCTTAT & 58 \\
\hline CD133 & CTGGGGCTGCTGTTTATTATTCTG & ACGCCTTGTCCTTGGTAGTGTTG & 61 \\
\hline$C D 44$ & GTGATCAACAGTGGCAATGG & GGGCCCTAATTTCAGAAAGC & 58 \\
\hline LGR5 & GAGGATCTGGTGAGCCTGAGAA & CATAAGTGATGCTGGAGCTGGTAA & 61 \\
\hline $\mathrm{HOl}$ & GGTGACCCGAGAGGGCTT & CGAAGACTGGGCTCTCCTTGT & 59 \\
\hline OLFM4 & TGGTGAACATCAGCAAACCG & TCCСТАCCCCAAGCACCATA & 58 \\
\hline ASCL2 & TGACCTGGGGCGTAATAAAG & AGTTCACGCTCCCTTGAAGA & 58 \\
\hline OCT4 & GACAACAATGAGAACCTTCAGGAGA & CTGGCGCCGGTTACAGAACCA & 59 \\
\hline$P S P$ & GGCGGGGCAGTGCCTTTCAAA & TGTTGGCTGCGTCTCATCAAAACC & 61 \\
\hline$B M I 1$ & GGAGACCAGCAAGTATTGTCCTTTTG & CATTGCTGCTGGGCATCGTAAG & 60 \\
\hline$M E L K$ & CTTGGATCAGAGGCAGATGTTTGGAG & GTTGTAATCTTGCATGATCCAGG & 60 \\
\hline TERT & CGGAAGAGTGTCTGGAGCAA & GGATGAAGCGGAGTCTGGA & 58 \\
\hline SOX2 & ACCGGCGGCAACCAGAAGAACAG & GCGCCGCGGCCGGTATTTAT & 60 \\
\hline SOX4 & ATTGATGTTGTTGTTGATGG & AAGCAAAATAAAACAAAACC & 58 \\
\hline SOX9 & AGGTGCTCAAAGGCTACGACT & AGATGTGCGTCTGCTCCGTG & 58 \\
\hline$D K K 1$ & СТTTCTCССТCTTGAGTCCTTCTG & CATAGCGTGACGCATGCAGCGTT & 60 \\
\hline WNT1 & CTGCCTCTCTTCTTCCCCTT & TCACAGCTGTTCAATGGCTC & 58 \\
\hline WNT5A & ССАССТТССТСТТСАСАСТG & CGAACAAGTAATGCCCTCTC & 59 \\
\hline WNT5B & CCGCCTCTGCAACAAGACCT & AACTTGCAGTGGCAGCGCTC & 58 \\
\hline$\beta$-catenin & TCTTGGCTATTACGACAG & ССТСТАТАССАСССАСТТ & 57 \\
\hline TCF1 & CTCATCACCGACACCACCAACC & TCCCACGAAGCAGCGACAGT & 60 \\
\hline TCF4 & GCCTCTCATCACGTACAGCA & GGATGGGGGATTTGTCCTAC & 59 \\
\hline LEF1 & CAGAGAAAGGAGCAGGAGCCAA & TGATGTCAGTGTTCCTTTGGCG & 60 \\
\hline$\beta$-actin & GACCACACCTTCTACAATGAG & GCATACCCCTCGTAGATGGG & 59 \\
\hline
\end{tabular}




\begin{tabular}{lccccc}
\hline SUPPLEMENTARY TABLE 2. List of Antibodies Used in Western Blotting & & & & \\
\hline Primary Antibodies & Supplier & Species & Type & Dilution & Reference \\
\hline Cytokeratin 18 & Abcam (Cambridge, UK) & Mouse & Monoclonal & $1: 2000$ & ab668 \\
EPCAM & Santa Cruz Biotechnology, Inc. (Dallas, Texas) & Mouse & Monoclonal & $1: 1000$ & sc-25308 \\
Vimentin & Abcam (Cambridge, UK) & Rabbit & Monoclonal & $1: 2000$ & ab92547 \\
E-Cadherin & Abcam (Cambridge, UK) & Mouse & Monoclonal & $1: 1000$ & ab1416 \\
Slug & Cell Signaling Technology (Danvers, Mass) & Rabbit & Monoclonal & $1: 1000$ & $\# 9585$ \\
Snail & Cell Signaling Technology (Danvers, Mass) & Rabbit & Monoclonal & $1: 1000$ & $\# 3879$ \\
HER1 & Abcam (Cambridge, UK) & Rabbit & Polyclonal & $1: 1000$ & ab131498 \\
HER2 & Abcam (Cambridge, UK) & Mouse & Monoclonal & $1: 1000$ & ab16901 \\
pERK1/2 & Cell Signaling Technology (Danvers, Mass) & Rabbit & Polyclonal & $1: 1000$ & $\# 9101$ \\
ERK1 & Abcam (Cambridge, UK) & Rabbit & Polyclonal & $1: 1000$ & ab137766 \\
ERK2 & Abcam (Cambridge, UK) & Rabbit & Monoclonal & $1: 1000$ & ab32081 \\
PTEN & Cell Signaling Technology (Danvers, Mass) & Rabbit & Polyclonal & $1: 1000$ & $\# 9552$ \\
SMAD4 & Abcam (Cambridge, UK) & Rabbit & Polyclonal & $1: 2000$ & ab137861 \\
$\beta$-actin & Santa Cruz Biotechnology, Inc. (Dallas, Texas) & Mouse & Monoclonal & $1: 500$ & sc-47778 \\
\hline
\end{tabular}




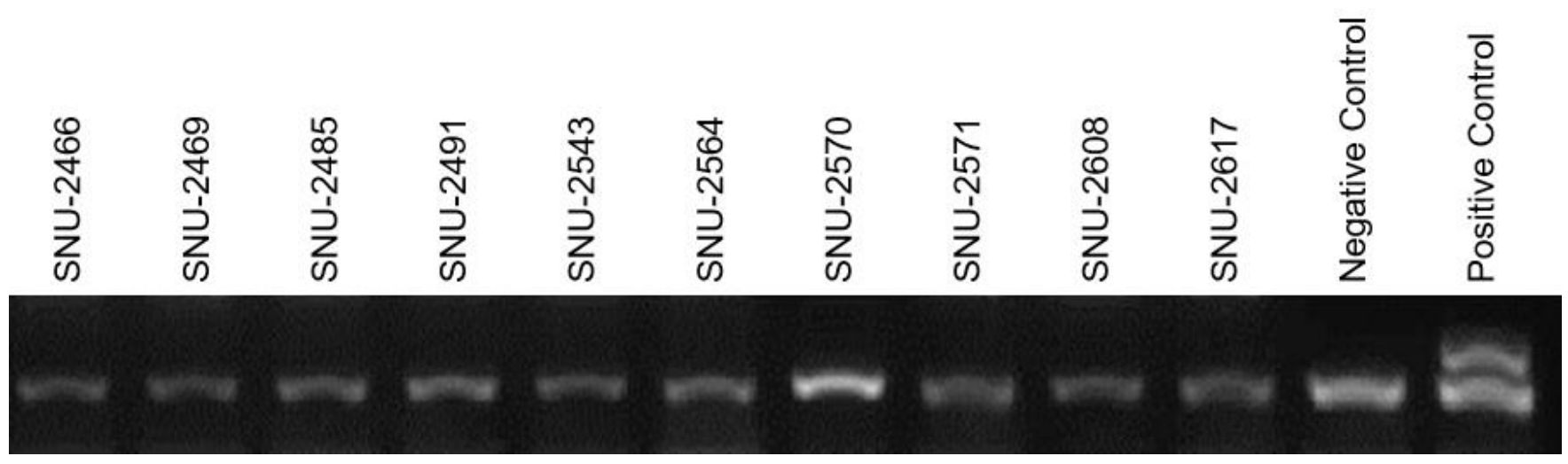

SUPPLEMENTARY FIGURE 1. Mycoplasma test of ten newly established pancreatic cancer cell lines. Mycoplasma contamination was tested by the 16S-rRNA-gene-based polymerase chain reaction (PCR) amplification. All cell lines were confirmed to be free of mycoplasma contamination. 


\begin{tabular}{|c|c|c|c|c|c|c|c|c|c|c|c|c|}
\hline \multicolumn{13}{|c|}{ SUPPLEMENTARY TABLE 3. Gene Lists for Further Sorting WES Data } \\
\hline$A B L 1$ & $B A P 1$ & $C C N D 1$ & COL1A1 & EP300 & FANCC & $G 6 P D$ & $I K B K E$ & $L C K$ & $M E T$ & NCOA2 & PAX8 & PPARG \\
\hline$A B L 2$ & $B C L 10$ & CCND2 & $C R B N$ & EP400 & FANCD2 & GATA1 & $I K Z F 1$ & LIFR & $M I T F$ & NCOA4 & PBRM1 & $P P P 2 R 1 A$ \\
\hline$A C V R 2 A$ & $B C L 11 A$ & CCNE1 & $C R E B 1$ & EPCAM & FANCE & GATA2 & $I L 2$ & LPHN3 & $M L H 1$ & $N F 1$ & $P B X 1$ & PRDM1 \\
\hline ADAMTS20 & $B C L 11 B$ & $C D 79 A$ & $C R E B B P$ & ЕРНA3 & FANCF & GATA3 & $I L 21 R$ & $L P P$ & $M L L$ & $N F 2$ & PDE4DIP & PRF1 \\
\hline$A F F 1$ & $B C L 2$ & $C D 79 B$ & $C R K L$ & EPHA7 & FANCG & $G D N F$ & $I L 6 S T$ & $L R P 1 B$ & $M L L 2$ & NFE2L2 & $P D G F B$ & PRKARIA \\
\hline$A F F 3$ & $B C L 2 L 1$ & $C D C 73$ & CRTC1 & EPHB1 & FANCI & GNA11 & $I L 7 R$ & $L T F$ & $M L L 3$ & $N F K B 1$ & PDGFRA & $P R K D C$ \\
\hline$A I P$ & $B C L 2 L 2$ & $\mathrm{CDH1}$ & CSF1R & EPHB4 & FANCL & $G N A Q$ & $I N G 4$ & $L T K$ & MLLT10 & $N F K B 2$ & $P D G F R B$ & PRSS1 \\
\hline$A K A P 9$ & $B C L 3$ & $C D H 11$ & CSMD3 & ЕРНB6 & FANCM & $G N A S$ & IRF4 & $M A F$ & $M M P 2$ & NIN & PER1 & $P S I P 1$ \\
\hline$A K T 1$ & $B C L 6$ & $\mathrm{CDH} 2$ & CTNNA1 & $E R B B 2$ & $F A S$ & $G P C 3$ & $I R S 2$ & $M A F B$ & $M N 1$ & $N K X 2-1$ & $P G A P 3$ & PTCH1 \\
\hline$A K T 2$ & $B C L 9$ & $\mathrm{CDH} 20$ & CTNNB1 & $E R B B 3$ & $F B X W 7$ & GPR124 & ITGA10 & $M A G E A 1$ & $M P L$ & $N L R P 1$ & $\mathrm{PHOX} 2 \mathrm{~B}$ & PTEN \\
\hline AKT3 & $B C R$ & CDH5 & CTRC & $E R B B 4$ & FGFRI & GRM8 & ITGA9 & $M A G I 1$ & MRE11A & NOTCH1 & $P I K 3 C 2 B$ & PTGS2 \\
\hline$A L K$ & $B I R C 2$ & $C D K 12$ & $C Y L D$ & ERCC1 & FGFR2 & GUCY1A2 & $I T G B 2$ & $M A L T 1$ & MSH2 & NOTCH2 & $P I K 3 C A$ & PTPN11 \\
\hline$A P C$ & $B I R C 3$ & $C D K 4$ & СYР2C19 & $E R C C 2$ & $F G F R 3$ & $H C A R 1$ & $I T G B 3$ & $M A M L 2$ & MSH6 & NOTCH4 & $P I K 3 C B$ & PTPRD \\
\hline$A R$ & BIRC5 & CDK6 & CYP2D6 & ERCC3 & FGFR4 & $H I F 1 A$ & $J A K 1$ & $M A P 2 K 1$ & MTOR & $N P M 1$ & PIK3CD & PTPRT \\
\hline ARIDIA & $B L M$ & $C D K 8$ & $D A X X$ & ERCC4 & $F H$ & $H L F$ & $J A K 2$ & $M A P 2 K 2$ & $M T R$ & $N R A S$ & $P I K 3 C G$ & $R A D 50$ \\
\hline ARID2 & $B L N K$ & $C D K N 1 C$ & $D C C$ & ERCC5 & $F L C N$ & $H N F 1 A$ & $J A K 3$ & $M A P 2 K 4$ & MTRR & NSD1 & $P I K 3 R 1$ & $R A D 51 C$ \\
\hline$A R N T$ & $B M P R 1 A$ & $C D K N 2 A$ & $D D B 2$ & $E R G$ & FLII & HOOK3 & $J U N$ & $M A P 3 K 7$ & $M U C 1$ & NTRK1 & $P I K 3 R 2$ & $R A D 51 D$ \\
\hline$A S X L 1$ & $B R A F$ & $C D K N 2 B$ & DDIT3 & ESR1 & FLT1 & $H R A S$ & KАT6A & $M A P K 1$ & MUTYH & NTRK3 & PIMI & $R A F 1$ \\
\hline$A T F 1$ & $B R C A 1$ & $C D K N 2 C$ & $D D R 2$ & ETS1 & FLT3 & HSP90AA1 & КАТбB & $M A P K 8$ & $M Y B$ & NUMA1 & PKHD1 & $R A L G D S$ \\
\hline ATM & $B R C A 2$ & CEBPA & $D E K$ & ETV1 & FLT4 & $H S P 90 A B 1$ & KDM5C & $M A R K 1$ & $M Y C$ & NUP214 & $P L A G 1$ & $R A R A$ \\
\hline$A T R$ & $B R D 3$ & CEP57 & DICERI & ETV4 & FN1 & $I C K$ & KDM6A & $M A R K 4$ & $M Y C L 1$ & NUP98 & $P L C G 1$ & $R B 1$ \\
\hline$A T R X$ & BRIP1 & CFTR & $D I S 3 L 2$ & $E X T 1$ & FOXL2 & $I D H 1$ & $K D R$ & $M A X$ & $M Y C N$ & $P A K 3$ & PLEKHG5 & RECQL4 \\
\hline$A U R K A$ & $B T K$ & CHEK1 & DNMT3A & $E X T 2$ & FOXO1 & $I D H 2$ & $K E A P 1$ & $M B D 1$ & MYD88 & PALB2 & $P M L$ & $R E L$ \\
\hline$A U R K B$ & $B U B 1 B$ & CHEK2 & $D P Y D$ & $E Z H 2$ & FOXO3 & $I G F I R$ & KIT & $M C L 1$ & MYH11 & $P A R P 1$ & PMS1 & $R E T$ \\
\hline$A U R K C$ & CARDI1 & $C I C$ & $D S T$ & $F A M 123 B$ & $F O X P 1$ & $I G F 2$ & $K L F 6$ & $M D M 2$ & МҮНЯ & $P A X 3$ & $P M S 2$ & $R H B D F 2$ \\
\hline$A X L$ & $C A S C 5$ & $C K S 1 B$ & $E G F R$ & $F A N C A$ & FOXP4 & $I G F 2 R$ & $K R A S$ & $M D M 4$ & $N B N$ & $P A X 5$ & POT1 & $\mathrm{RHOH}$ \\
\hline$B A I 3$ & $C B L$ & CMPK1 & $E M L 4$ & $F A N C B$ & FZR1 & $I K B K B$ & $L A M P 1$ & MEN1 & NCOA1 & $P A X 7$ & POU5F1 & RNASEL \\
\hline
\end{tabular}

\begin{tabular}{l|l} 
www.pancreasjournal.com & $\mathbf{4}$
\end{tabular} 


\begin{tabular}{|c|c|c|c|c|c|c|c|c|c|c|}
\hline \multicolumn{11}{|c|}{ SUPPLEMENTARY TABLE 3. (Continued) } \\
\hline$R N F 2$ & $S B D S$ & $S F 3 B 1$ & $S M O$ & STK11 & $T B X 22$ & TFE3 & TNFAIP3 & TRIPII & $V H L$ & $X R C C 2$ \\
\hline RNF213 & SDHA & SGK1 & $S M U G 1$ & STK36 & TCF12 & $T G F B R 2$ & TNFRSF14 & TRRAP & WAS & ZNF384 \\
\hline ROS1 & SDHAF2 & SH2D1A & SOCS1 & SUFU & $T C F 3$ & $T G M 7$ & $T N K 2$ & $T S C 1$ & WHSCl & ZNF521 \\
\hline RPS6KA2 & $S D H B$ & SLX4 & SOX11 & SYK & $T C F 7 L 1$ & THBS1 & TOP1 & $T S C 2$ & $W R N$ & \\
\hline$R R M I$ & $S D H C$ & $S M A D 2$ & $S O X 2$ & SYNE1 & TCF7L2 & TIMP3 & TP53 & TSHR & $W T 1$ & \\
\hline$R U N X 1$ & $S D H D$ & SMAD4 & SPINK1 & TAF1 & TCL1A & $T L R 4$ & $T P R$ & UBR5 & $X P A$ & \\
\hline$R U N X 1 T 1$ & SEPTQ & SMARCA4 & $S R C$ & $T A F 1 L$ & TET1 & $T L X 1$ & TRIM24 & UGTIAI & $X P C$ & \\
\hline SAMD9 & SETD2 & $S M A R C B 1$ & $S S X 1$ & TAL1 & TET2 & TMEM127 & TRIM33 & USP9X & XPO1 & \\
\hline
\end{tabular}




\begin{tabular}{|c|c|c|c|c|}
\hline \multicolumn{5}{|c|}{ SUPPLEMENTARY TABLE 4. Mutational Status of Selected Genes } \\
\hline Cell Line & Gene & Mutation & Clinical Significance & Reference \\
\hline \multirow[t]{10}{*}{ SNU-2466 } & BRCAl & c. $3113 \mathrm{~A}>$ G/p.Glu1038Gly & Uncertain significance & 42 \\
\hline & & c. $2612 \mathrm{C}>\mathrm{T} / \mathrm{p}$. Pro871Leu & Uncertain significance & 43 \\
\hline & MSH2 & c. $1168 \mathrm{C}>\mathrm{T} / \mathrm{p}$. Leu390Phe & Uncertain significance & 42 \\
\hline & $K R A S$ & c. $35 \mathrm{G}>\mathrm{A}$ & Pathogenic/Likely pathogenic & 44 \\
\hline & TP53 & c. $503 \mathrm{~A}>\mathrm{G} / \mathrm{p}$. His168Arg & Likely pathogenic & 45 \\
\hline & & c. $215 \mathrm{C}>\mathrm{G} / \mathrm{p}$. Pro72Arg & drug response & 46 \\
\hline & PREX2 & c. $4463 \mathrm{C}>\mathrm{T} / \mathrm{p}$. Ser1488Leu & NA & NA \\
\hline & $C D K N 2 A$ & c.51_59delCACGGCCGC/p.Thr18_Ala20del & Pathogenic/Likely pathogenic & 47 \\
\hline & $A C V R 1 B$ & c. $1180 \mathrm{G}>\mathrm{A} / \mathrm{p}$. Asp394Asn & Pathogenic/Likely pathogenic & 48 \\
\hline & SMARCA2 & c. $4638 \mathrm{C}>\mathrm{G} / \mathrm{p}$. Asp $1546 \mathrm{Glu}$ & Likely pathogenic & 49 \\
\hline \multirow[t]{12}{*}{ SNU-2469 } & $P A L B 2$ & c. $1676 \mathrm{~A}>\mathrm{G} / \mathrm{p} . \mathrm{G} \ln 559 \mathrm{Arg}$ & Likely benign & 50 \\
\hline & $B R C A 1$ & c. $4900 \mathrm{~A}>\mathrm{G} / \mathrm{p}$. Ser1634Gly & Likely benign & 42 \\
\hline & & c. $3113 \mathrm{~A}>$ G/p.Glu1038Gly & Uncertain significance & 42 \\
\hline & & c. $2612 \mathrm{C}>\mathrm{T} / \mathrm{p}$. Pro871Leu & Uncertain significance & 43 \\
\hline & $K R A S$ & c. $35 \mathrm{G}>\mathrm{A} / \mathrm{p} . \mathrm{Gly} 12 \mathrm{Asp}$ & Pathogenic & 44 \\
\hline & TP53 & c.982_983insA/p.Phe328fs & Likely pathogenic & 51 \\
\hline & PREX2 & c. $4463 \mathrm{C}>\mathrm{T} / \mathrm{p}$. Ser1488Leu & NA & 52 \\
\hline & SMAD4 & c. $.513 \mathrm{G}>\mathrm{C} / \mathrm{p} . \mathrm{Glu} 171 \mathrm{Asp}$ & NA & - \\
\hline & & c. $1523 \mathrm{G}>\mathrm{A} / \mathrm{p} . \mathrm{Gly} 508 \mathrm{Asp}$ & NA & - \\
\hline & PIK3R3 & c. $1088 \mathrm{~A}>\mathrm{G} / \mathrm{p}$. Asn363Ser & unknown & - \\
\hline & & c.987T>A/p.Asn329Lys & Likely benign & 53 \\
\hline & $N O V$ & c. $125 \mathrm{G}>\mathrm{A} / \mathrm{p} . \operatorname{Arg} 42 \mathrm{Gln}$ & Pathogenic & 54 \\
\hline \multirow[t]{9}{*}{ SNU-2485 } & $A T M$ & c. $215 \mathrm{~T}>\mathrm{C} / \mathrm{p}$. Leu72Pro & Likely benign & LMM Criteria \\
\hline & $B R C A 2$ & c. $865 \mathrm{~A}>\mathrm{C} / \mathrm{p} . \mathrm{Asn} 289 \mathrm{His}$ & $\begin{array}{l}\text { Conflicting interpretations of } \\
\text { pathogenicity }\end{array}$ & 55 \\
\hline & $P A L B 2$ & c.3226C $>$ T/p.His1076Tyr & Uncertain significance & 42 \\
\hline & $K R A S$ & c. $35 \mathrm{G}>\mathrm{A} / \mathrm{p}$. Gly $12 \mathrm{Asp}$ & Pathogenic & 44 \\
\hline & TP53 & c. $560-1 \mathrm{G}>\mathrm{A}$ & Pathogenic & $\begin{array}{c}\text { Mayo Clinic Genetic Testing } \\
\text { Laboratories }\end{array}$ \\
\hline & PREX2 & c. $4463 \mathrm{C}>\mathrm{T} / \mathrm{p}$. Ser1488Leu & NA & 52 \\
\hline & $C D K N 2 A$ & c.208_213delCCCAAC/p.Pro70_Asn71del & Pathogenic/Likely pathogenic & 47 \\
\hline & SMARCA2 & c. $4638 \mathrm{C}>\mathrm{G} / \mathrm{p}$. Asp1546Glu & Likely pathogenic & 49 \\
\hline & GATA6 & c. $43 \mathrm{G}>$ C/p.Gly $15 \mathrm{Arg}$ & likely benign & 42 \\
\hline \multirow[t]{4}{*}{ SNU-2491 } & $B R C A 2$ & c. $1114 \mathrm{~A}>\mathrm{C} / \mathrm{p} . \mathrm{Asn} 372 \mathrm{His}$ & Likely pathogenic & 56 \\
\hline & $P A L B 2$ & c. $1676 A>$ G/p.Gln559Arg & Likely benign & 50 \\
\hline & MLH1 & c.649C $>$ T/p.Arg217Cys & Uncertain significance & 57 \\
\hline & $K R A S$ & c. $35 \mathrm{G}>\mathrm{A} / \mathrm{p}$. Gly $12 \mathrm{Asp}$ & Pathogenic & 44 \\
\hline
\end{tabular}




\begin{tabular}{|c|c|c|c|c|}
\hline \multicolumn{5}{|c|}{ SUPPLEMENTARY TABLE 4. (Continued) } \\
\hline Cell Line & Gene & Mutation & Clinical Significance & Reference \\
\hline \multirow{15}{*}{ SNU-2543 } & TP53 & c. $733 \mathrm{G}>\mathrm{A}$ & protein_protein_contact & - \\
\hline & & c. $215 \mathrm{C}>\mathrm{G} / \mathrm{p}$. Pro72Arg & drug response & 46 \\
\hline & SMARCA2 & c. $4638 \mathrm{C}>\mathrm{G} / \mathrm{p}$. Asp $1546 \mathrm{Glu}$ & Likely pathogenic & 49 \\
\hline & ARIDIA & c.5548dupG/p.Asp1850fs & Pathogenic & 58 \\
\hline & $A R I D I B$ & c.363_380delACAGCAGCAGCAGCAGCA/p.Gln122_Gln127del & Pathogenic & 59 \\
\hline & PIK3R3 & c. $987 \mathrm{~T}>\mathrm{A} / \mathrm{p}$. Asn329Lys & Likely benign & 53 \\
\hline & $E R B B 2$ & c. $3508 \mathrm{C}>$ G/p.Pro1170Ala & not provided & 60 \\
\hline & BRCA2 & c. $1114 \mathrm{~A}>\mathrm{C} / \mathrm{p}$. Asn372His & Likely pathogenic & 56 \\
\hline & $B R C A 1$ & c. $4900 \mathrm{~A}>\mathrm{G} / \mathrm{p}$. Ser1634Gly & Likely benign & 42 \\
\hline & & c. $3113 \mathrm{~A}>$ G/p.Glu1038Gly & Uncertain significance & 42 \\
\hline & & c. $2612 \mathrm{C}>\mathrm{T} / \mathrm{p}$. Pro871Leu & Uncertain significance & 43 \\
\hline & RPAl & c. $1848 \mathrm{G}>\mathrm{T} / \mathrm{p}$. Met616Ile & $\mathrm{NA}$ & NA \\
\hline & $K R A S$ & c.34G>T/p.Gly12Cys & Pathogenic & 61 \\
\hline & TP53 & c. $993+2 \mathrm{~T}>\mathrm{A}$ & Pathogenic & NA \\
\hline & & c. $215 \mathrm{C}>\mathrm{G} / \mathrm{p}$. Pro72Arg & drug response & 46 \\
\hline \multirow{8}{*}{ SNU-2564 } & PREX2 & c. $4463 \mathrm{C}>\mathrm{T} / \mathrm{p}$. Ser1488Leu & NA & 52 \\
\hline & SMARCA2 & c. $4638 \mathrm{C}>$ G/p.Asp1546Glu & Likely pathogenic & 49 \\
\hline & ARIDIA & c. $148 \mathrm{~A}>\mathrm{G} / \mathrm{p}$. Met50Val & Uncertain significance & NA \\
\hline & $P A L B 2$ & c. $1676 A>$ G/p.Gln559Arg & Likely benign & 50 \\
\hline & $K R A S$ & c. $35 \mathrm{G}>\mathrm{A} / \mathrm{p}$. Gly $12 \mathrm{Asp}$ & Pathogenic & 44 \\
\hline & RNF43 & c. $1093 \mathrm{G}>\mathrm{A} / \mathrm{p}$. Ala365Thr & NA & - \\
\hline & SLIT2 & c. $4527 \mathrm{G}>\mathrm{T} / \mathrm{p} . \operatorname{Arg} 1509 \mathrm{Ser}$ & - & 62,63 \\
\hline & $P I K 3 R 3$ & c. $987 \mathrm{~T}>\mathrm{A} / \mathrm{p}$. Asn329Lys & Pathogenic & 64 \\
\hline \multirow{13}{*}{ SNU-2570 } & $E R B B 2$ & c. $3508 \mathrm{C}>\mathrm{G} / \mathrm{p}$. Pro1170Ala & With untested allele & 60 \\
\hline & NOV & c. $125 \mathrm{G}>\mathrm{A} / \mathrm{p}$. Arg42Gln & NA & - \\
\hline & $B R C A 1$ & c. $4900 \mathrm{~A}>\mathrm{G} / \mathrm{p}$. Ser1634Gly & Likely benign & 42 \\
\hline & & c. $3113 \mathrm{~A}>$ G/p.Glu1038Gly & Uncertain significance & 42. \\
\hline & & c. $2612 \mathrm{C}>\mathrm{T} / \mathrm{p}$. Pro871Leu & Uncertain significance & 43 \\
\hline & MSH2 & c. $1168 \mathrm{C}>\mathrm{T} / \mathrm{p}$. Leu390Phe & Uncertain significance & 42 \\
\hline & $K R A S$ & c. $35 \mathrm{G}>\mathrm{A} / \mathrm{p} . \mathrm{Gly} 12 \mathrm{Asp}$ & Pathogenic & 44 \\
\hline & TP53 & c. $215 \mathrm{C}>\mathrm{G} / \mathrm{p}$. Pro72Arg & drug response & 46 \\
\hline & PREX2 & c. $4463 \mathrm{C}>\mathrm{T} / \mathrm{p}$. Ser1488Leu & NA & 52 \\
\hline & $A C V R 1 B$ & c. $1328 \mathrm{~A}>\mathrm{T} / \mathrm{p}$. Asp443Val & NA & - \\
\hline & $T G F B R 2$ & c. $487 \mathrm{~T}>\mathrm{C} / \mathrm{p}$. Cys $163 \mathrm{Arg}$ & $\begin{array}{l}\text { Conflicting interpretations of } \\
\text { pathogenicity }\end{array}$ & - \\
\hline & $P I K 3 R 3$ & c.987T>A/p.Asn329Lys & Pathogenic & 64 \\
\hline & $E R B B 2$ & c. $3508 \mathrm{C}>$ G/p.Pro1170Ala & With untested allele & - \\
\hline
\end{tabular}




\begin{tabular}{|c|c|c|c|c|}
\hline \multicolumn{5}{|c|}{ SUPPLEMENTARY TABLE 4. (Continued) } \\
\hline Cell Line & Gene & Mutation & Clinical Significance & Reference \\
\hline \multirow[t]{7}{*}{ SNU-2571 } & $P A L B 2$ & c. $1676 \mathrm{~A}>\mathrm{G} / \mathrm{p} . \mathrm{Gln} 559 \mathrm{Arg}$ & Likely benign & 50 \\
\hline & $K R A S$ & c. $35 \mathrm{G}>\mathrm{A} / \mathrm{p} . \mathrm{Gly} 12 \mathrm{Asp}$ & Pathogenic & 44 \\
\hline & TP53 & c. $215 \mathrm{C}>\mathrm{G} / \mathrm{p}$. Pro72Arg & drug response & 46 \\
\hline & & c.695T $>$ G/p.Ile232Ser & Uncertain significance & - \\
\hline & ARIDIA & c. $4703 \mathrm{delC} / \mathrm{p}$. Pro $1568 \mathrm{fs}$ & frame shift & NA \\
\hline & $P I K 3 R 3$ & c. $987 \mathrm{~T}>\mathrm{A} / \mathrm{p}$. Asn329Lys & Pathogenic & 64 \\
\hline & $E R B B 2$ & c.3508C $>$ G/p.Pro1170Ala & With untested allele & \\
\hline \multirow[t]{5}{*}{ SNU-2608 } & $B R C A 2$ & c.3220A > T/p.Ser1074Cys & NA & - \\
\hline & $K R A S$ & c. $35 \mathrm{G}>\mathrm{A} / \mathrm{p}$. Gly $12 \mathrm{Asp}$ & Pathogenic & 44 \\
\hline & SMARCA2 & c. $4247 \mathrm{G}>$ C/p.Gly1416Ala & NA & 49 \\
\hline & ROBO1 & c. $2936 \mathrm{C}>\mathrm{T} / \mathrm{p} . \mathrm{Thr} 979 \mathrm{Met}$ & - & 63 \\
\hline & PIK3R3 & c. $987 \mathrm{~T}>\mathrm{A} / \mathrm{p}$. Asn329Lys & Pathogenic & - \\
\hline \multirow[t]{10}{*}{ SNU-2617 } & $K R A S$ & c. $35 \mathrm{G}>\mathrm{A} / \mathrm{p} . \mathrm{Gly} 12 \mathrm{Asp}$ & Pathogenic & 44 \\
\hline & TP53 & c. $853 \mathrm{G}>\mathrm{A}$ & $\begin{array}{l}\text { Likely pathogenic, protein- } \\
\text { protein interaction }\end{array}$ & - \\
\hline & PREX2 & c.4489G >A/p.Ala1497Thr & NA & - \\
\hline & $S M A R C A 2$ & c. $4638 \mathrm{C}>\mathrm{G} / \mathrm{p}$. Asp $1546 \mathrm{Glu}$ & Likely benign & 65 \\
\hline & SLIT2 & c. $3259 \mathrm{G}>$ C/p.Asp1087His & NA & 62 \\
\hline & $P I K 3 R 3$ & c.987T>A/p.Asn329Lys & Pathogenic & - \\
\hline & $E R B B 2$ & c.3508C >G/p.Pro1170Ala & With untested allele & - \\
\hline & & c.1963A>G/p.Ile655Val & not specified & 60 . \\
\hline & $N O V$ & c. $125 \mathrm{G}>\mathrm{A} / \mathrm{p}$. Arg $42 \mathrm{Gln}$ & NA & - \\
\hline & GATA6 & c. $43 \mathrm{G}>$ C/p.Gly15Arg & Likely benign & \\
\hline
\end{tabular}




\begin{tabular}{|c|c|c|c|}
\hline Gene & Mutation & RS Number & ClinVar Significance \\
\hline Clorf177 & c. $1028 \mathrm{G}>\mathrm{A} / \mathrm{p}$. Cys $343 \mathrm{Tyr}$ & rs75191355 & NA \\
\hline Clorf177 & c. $1228 \mathrm{G}>\mathrm{T} / \mathrm{p}$.Gly410Cys & rs74642512 & NA \\
\hline$N G F$ & c. $104 \mathrm{C}>\mathrm{T} / \mathrm{p}$. Ala35Val & rs6330 & Benign \\
\hline MYL1 & c. $1 \mathrm{delA} / \mathrm{p}$. Met1fs & - & - \\
\hline CCSERI & c.1940T >C/p.Leu647Ser & rs62314392 & NA \\
\hline$W W C 2$ & c. $2782 \mathrm{G}>$ C/p.Asp928His & rs3814422 & NA \\
\hline$A P B A 1$ & c. $550 \mathrm{~T}>\mathrm{G} / \mathrm{p} . \operatorname{Ser} 184 \mathrm{Ala}$ & rs34788368 & NA \\
\hline$P A L D 1$ & c. $422 \mathrm{C}>\mathrm{T} / \mathrm{p}$. Ser141Leu & rs 2275060 & NA \\
\hline VPS11 & c. $2663 \mathrm{~A}>\mathrm{G} / \mathrm{p} . \mathrm{Lys} 888 \mathrm{Arg}$ & rs 15818 & NA \\
\hline PCSK 4 & c. $823 \mathrm{G}>\mathrm{A} / \mathrm{p}$. Ala275Thr & rs4807119 & NA \\
\hline NLRP12 & c. $116 \mathrm{G}>\mathrm{T} / \mathrm{p} . \mathrm{Gly} 39 \mathrm{Val}$ & rs34436714 & Benign \\
\hline B4GALT5 & c. $181 \mathrm{G}>\mathrm{A} / \mathrm{p}$. Gly61Ser & rs2273086 & NA \\
\hline
\end{tabular}

